\title{
Cognitive Strategies of Behavior of Primary School Children in the Digital Environment
}

\author{
Irina S. Luchinkina * \\ Crimean Engineering and Pedagogical University named after Fevzi Yakubov, 295015, Simferopol \\ (Russia),8Uchebnyjlane,miss_luchinkina@mail.ru
}

\begin{abstract}
The article discusses the problem of the influence of digital space and digital environment on the cognitive strategies of primary school students. The features of the personality included in the digital space are given and theoretically analyzed. The contradictions in the concept of a "digital identity" are noted.

The study involved 95 primary school children, 53 of whom were active users of the digital environment; and 42 were passive users. The following methods were used in the study: expert assessment, questionnaires, and psychological tests. The empirical study revealed significant differences in such parameters as attentiveness, statistical (re)presentation, development of imagination, the ability to linearly distinguish and draw the inference from linear relationships and the ability to respond quickly. Unlike students with passive digital behavior, primary school students with active digital behavior have a high level of responsiveness to changing conditions. It is noted that the number of elements in the proposed experimental problems negatively affects the speed with which primary school students with active digital behavior troubleshoot. The study also revealed levels of abstraction and dynamic synthesis, including the ability to determine the development of dynamic series in sample groups quantitatively.

Empirically revealed differences between respondents in terms of cognitive strategies include: impulsivity, reflexivity, flexibility of thinking and rigidity. Studies (showed the presence of) revealed typologies of primary school students with active and passive behavior in digital environment, depending on the parameters of thinking and cognitive processes in general.
\end{abstract}

Keywords: digital behavior, primary school children, cognitive styles, digital activity, digital environment.

\section{(C) 2020 Irina S. Luchinkina}

This is an open access article distributed under the terms of the Creative Commons Attribution License (CC BY 4.0), which permits unrestricted use, distribution, and reproduction in any medium, provided the original author and source are credited.

Published by Kazan federal university and peer-reviewed under responsibility of IFTE-2020 (VI International Forum on Teacher Education)

\footnotetext{
* Corresponding author. E-mail: miss_luchinkina@mail.ru
} 


\section{Introduction}

The postmodern world is rapidly developing, raising a tsunami of information that cannot be digested by the human brain which leads to destruction of memory barriers and perception. In the desire to cover all flows of information, a person who aspires to multitasking is immersed in information and digital diversity (Taylor, 2006). As a result, distorted cognitive processes arise that lead to a passive perception of information or difficulties in processing this information.

Clip thinking has become the main metaphor of the last decade (Mityagina \& Dolgopolova, 2009). The passion for gadgets and the desire for a more vivid image find its expression in digital environment, which represent a combination of technical achievements, virtual platforms and socio-cultural reality based on them (Zajceva, 2015; Nikulina, 2018). At the present time, there is almost no empirical material that fully reveals and explains the problem of cognitive strategies of a personality's digital behavior.

\section{Purpose and objectives of the study}

The purpose of the study is to analyse the cognitive strategies of active and passive users in the digital environment.

\section{Literature review}

Stefaneas and Vandoulakis (2012) concluded that digital space had contributed to the formation of new cognitive formation. The usual system of proof is mathematical. As a rule, in the mathematical practice, proof points to the logic of an utterance are based on the previously defined logics of other utterances.

However, the digital environment is characterized by evidence in the form of the concept of "proof-event". According to the authors, the logic of statements is not considered in general terms, but in relation to a specific situation. In this case, the proofs include social, communicative factors, as well as features of interaction in the semantic "proving-interpreter" field.

An interesting research on communication processes in the digital environment and their impact on decision-making has been conducted (Bondarenko, 2002). The author writes that in contrast to the communicative methods in real space ("one with one" or "one with many"), the communicative principle "many with many" operates in the digital environment. This principle, which resembles brainstorming, encourages the intensification of research work. The speed with which the dilemma can be resolved with the usage of the digital environment is unattainable in real-life conditions. 
Brainstorming in the digital environment leads to success in the process of combination of the abilities and knowledge of its users (Stefaneas \& Vandoulakis, 2012). Different users are specialists in distinct fields and can use a variety of scientific languages, which allows them not only to combine research values and standards but also to synthesize research methodologies and styles. As a result, according to researchers, their (personal) culture and skills are formed and developed.

Small and Vortan (2009) note that despite a variety of technologies and information that humans receive with its help, people are able to successfully get used to the digital space and to adapt their mental functions to it. Moreover, the modern human brain continues to evolve (mindfulness is increasing; consciousness is beginning to respond to all signals faster). As a result, thinking copes with many tasks much more effectively than it did before.

According to Lombina (2018), active involvement in the digital environment affects the brain activity, contributes to higher mental processes and, as a result, has an impact on the personality as a whole. For example, a child who is not used to receiving visual information has difficulties with the understanding of text and speech. The researcher notes that, primarily, the processes of perception suffer, and further problems with thinking can be noted.

In contrast to Lombina (2018), Small and Vortan (2009) note that modern children are already active digital users and the digital environment can affect them in different ways. On the one hand, it can lead to formation of the passive thinking and perception. On the other hand, it contributes to the development and stimulation of the new cognitive formation (Small \& Vortan, 2009). Moreover, classical teachers have repeatedly noted that, in principle, any socializing space is ambivalent.

Compernolle (2014) writes that a child of preschool age in a digital diversity becomes dependent on sounds, colours, movements of images and games. According to the author's point of view, this dependence is supported by a small release of dopamine that occurs when a virtual stimulus is presented. These conclusions are important in the context of the study of psychophysiological changes under the influence of the digital environment. However, it is necessary to clarify a number of nuances. Firstly, the increased need for a preschooler in the sound, colour and movement of the picture is fully justified by its age features. With the help of these reinforcements, the attention and cognitive activity of the child are formed.

Secondly, virtual children's games are usually designed for a certain age and level of development (in Google Play and AppStore: "Colors and shapes", "Children's puzzles", "Coloring Books for children", "Lesson: music and drawing", etc.). These games are both entertaining and educational in nature. Third, this research relates more to virtual space rather than to the digital one. 


\section{Methodology}

The study involved 95 primary school children, 53 of whom were active users of the digital environment; and 42 were passive users.

The following methods were used in the study: expert assessment, questionnaires, and psychological tests. Among the methods of psychodiagnostics there are progressive matrices of J. Raven; J. Kagan's method "Comparison of similar drawings"; J. Stroop's "Word-color interference" technique; M. A. Kholodnaya's "Ideal computer" technique.

At the first stage, according to the opinions of experts and the results of psychological surveys, groups of users with active and passive digital behavior were identified. At the second stage, we studied the features of thinking and cognitive strategies of primary school children with active and passive digital behavior.

\section{Results}

According to experts (psychologists with at least 5 years of experience in this area) and the results of the survey, active and passive users of the digital environment were identified. As a rule, active users of the digital environment spend about $60 \%$ of their time in the digital space: from virtual games to virtual learning.

\section{The results of progressive matrices (Raven, 2000)}

Thus, the level of attentiveness, statistical representation, and imagination among respondents in the group with passive digital behavior (77\%) exceeds the level of the same processes among respondents in the group with active digital behavior (43\%). The comparison of percentages using the Fischer test showed that the differences are significant: $77 \% / 43 \%, \varphi$ critical $=2.9, p \leq 0.01$.

The ability to distinguish linearly and infer from linear relationships is approximately the same in two sample groups (73\% inactive users and $69 \%$ active users). It should be noted that the ability to quickly respond to changing conditions was higher in the group of respondents with active digital behavior. The comparison of percentages using the Fischer test showed that the differences are significant: $84 \% / 52 \%, \varphi$ critical $=2.9, \mathrm{p} \leq 0.01$.

The dependence of the speed of solving problems on the number of elements in them was found in all groups of the sample. However, for passive users the number of elements is a factor that either complicates or simplifies the task. The situation is different for active respondents. The more elements there are in the proposed tasks, the less rapid their solution by respondents with active digital behavior $(\mathrm{p} \leq 0.05)$. 
A high level of abstraction and dynamic synthesis, including the ability to quantitatively and qualitatively determine the development of dynamic series, is more common among respondents with passive digital behavior. The comparison of percentages using the Fischer test showed that the differences were significant: $61 \% / 42 \%, \varphi$ critical $=2.9, \mathrm{p} \leq 0.01$.

The results of the study also revealed that students with active digital behavior can be characterized by a higher rate of fatigue and the onset of satiation of material in the process of solving problems, than students with passive digital behavior. There are also significant differences in the strategy of searching for correct answers: for respondents with active digital behavior a chaotic search strategy is more typical, whereas for respondents with passive digital behavior, the trial and error method is more peculiar.

The study of cognitive strategies impulsivity/reflexivity showed that respondents with passive digital behavior are more inherent to have a reflexive type of thinking $(p \leq 0.05)$. The reflexive type of thinking is expressed in the thoroughness of studying the instructions. Thus, J. Kagan noted that such respondents are characterized by a large oculomotor activity in the process of completing the task (Raven, 2000). It is worth mentioning that during the study, a group of schoolchildren with passive digital behavior more often fixed their eyes on similar elements of the drawing, comparing them in detail.

Reflexive respondents, as a rule, have such features as a well-developed meta-memory (introspective knowledge of the features of their own memory processes), the search and usage of the most productive methods for solving tasks.

Respondents with active digital behavior are more likely to have an impulsive type of thinking $(\mathrm{p} \leq 0.05)$. As a rule, decisions are made quickly and not always correctly. Students of this group most often demonstrate a superficial study of the instructions. In contrast to inactive users of the digital environment, active users have a less mature level of speech, which is also expressed in difficult self-regulation and control of their motor skills ( $\mathrm{p} \leq 0.05$ ).

The study also revealed that respondents with passive digital behavior are more likely to have flexible thinking with self-sufficient automation of cognitive functions and objective thinking, as opposed to a group of inactive users. However, atypical strategies were found in the sample group $(\mathrm{p} \leq 0.05)$.

Using the cluster analysis, several groups of primary school children with active and passive digital behavior were identified, depending on the features of their cognitive styles and measures of openness of the cognitive position: 
1) A group of primary school children with active digital behavior, can be characterized by an impulsive cognitive style, which is manifested in rapid decision-making. As a rule, this cognitive style is characterized by a large number of mistakes made by respondents. However, the study found that respondents in this group most often showed accurate responses. It was also revealed that among the respondents of this group, such indicators as flexible control with sufficient automation of cognitive functions and categorical thinking are expressed.

2) A group of primary school children with active digital behavior who have an impulsive cognitive style that manifests itself in the rapid and often incorrect decision-making. The respondents of this group are characterized by the rigid control with weak automation of cognitive functions and categorical thinking.

3) A group of primary school children with passive digital behavior, which is characterized by a reflective cognitive style, which is expressed in a slower pace of decision-making. As a rule, this cognitive style is characterized by a small number of mistakes made by respondents. However, the study revealed $61 \%$ of respondents who allowed incorrect answers. In addition, the respondents of this group are characterized by flexible control with sufficient automation of cognitive functions and an objectified type of thinking.

4) A group of primary school children with inactive digital behavior who are characterized by a reflective cognitive style, which is expressed in a slower pace of decision-making with subsequent correct responses. It was found that among the respondents of this group, such indicators as flexible control with sufficient automation of cognitive functions and categorical thinking are expressed.

\section{Discussions}

The ambiguity of the influence of the digital environment on the cognitive strategies of personality was discovered. The results of the study indicate that the issue of the digital environment and its impact on the personality of a primary school students and the personality as a whole needs further studies. Currently, there is not and cannot be a radical "bad" or radical "good" opinion when explaining the impact of the digital environment on the cognitive sphere of a child. Thus, the results of the study allowed us to determine that there are no significant differences between the active and passive groups of the sample based on the parameter of the ability to make linear distinctions and inference based on linear relationships. 
There are some differences. For example, the parameters of the level of attentiveness, statistical representation and imagination, abstraction and dynamic synthesis are higher among passive digital users. However, in terms of the ability to quickly respond to changing conditions, respondents with active digital behavior already show higher results.

The results indicate that the digital environment has an ambivalent effect on the cognitive strategies of a child. On the one hand, some cognitive strategies develop more slowly and intensively, on the other hand, others are strengthened, and perhaps new ones are formed.

The digital environment, including the Internet and other technical realities, becomes an environment of an individual's (personal) socialization. The ambiguity of the influence of digital reality on individuals is manifested in the fact that new digitalization capabilities (speed and brightness of information, its portioned presentation and, therefore, the transformation of the main activities (communication, education, games, creativity, recreation, professional and educational activities) leads to both personal growth and personal deviation. The active development of the society presupposes the uncertainty of the value system, an unstable culture, which complicates the socialization of an individual and destroys the existing ideas about this process.

The problem is that attempts to transfer the model of socialization to the digital environment make it impossible to understand the psychological patterns of digital socialization of an individual as a whole. The need for scientific research is to identify the psychological laws of socialization in the digital environment, which will reveal not only the characteristics of the digital personality, but will also highlight the stages by which this process unfolds.

\section{Conclusion}

School children with passive digital behavior are more likely to have a high level of attentiveness, statistical representation and development of imagination, the ability to linearly distinguish and make conclusions based on linear relationships, a high level of abstraction and dynamic synthesis, as well as reflexivity and the flexible control with sufficient automation of cognitive functions and the objectified type of thinking $(\mathrm{p} \leq 0,05)$.

School children with active digital behavior tend to have the ability to linearly distinguish and draw the inference based on linear relationships, the ability to respond quickly to changing conditions, the rigid control with poor automation of cognitive functions and categorical thinking $(p \leq 0,05)$. It is worth noting that atypical cognitive strategies were noted revealed among either of the sample groups $(p \leq 0,05)$. 
Thus, depending on the activity in the digital environment, primary school children may have different intellectual indicators, cognitive styles, and types of thinking in general. Modernization and the upgrade high-quality processing of new effective tools for the prevention and correction of cognitive impairment in school children with various types of digital activity is necessary.

\section{Acknowledgements}

The work is performed according to the Student Psychological Service Project Program.

\section{References}

Bondarenko, S. V. (2002). The social system of cyberspace as a new social community. Nauchnaya mysl' Kavkaza. Prilozhenie, 12(38), 32-39.

Compernolle, T. (2014). BrainChains: Discover your brain, to unleash its full potential in a hyperconnected, multitasking world. Amsterdam: Compublications.

Lombina, T. N. (2018). The features of teaching children with mosaic thinking. Obshchestvo: sociologiya, psihologiya, pedagogika, 1, 45-40.

Mityagina, E. V., \& Dolgopolova, N. S. (2009). "Clip consciousness" of youth in the modern information society. Vestnik Nizhegorodskogo universiteta, 3(15), 53-39.

Nikulina, T. V. (2018). Information and digital technologies in education: concepts, technologies, management. Pedagogicheskoe obrazovanie v Rossii, 8, 107-113.

Raven, J. (2000). The Raven's Progressive Matrices: Change and stability over culture and time. Cognitive Psychology, 41, 1-48.

Small, G., \& Vorgan G. (2009). iBrain: Surviving the Technological Alteration of the Modern Mind. New York: William Morrow Paperbacks.

Stefaneas, P., \& Vandoulakis, I. M. (2012). The web as a tool for proving. Metaphilosophy, 4(43), 480-498.

Taylor, L.M. (2006). Generation NeXt Comes to College: 2006 Updates and Emerging Issues. A Collection of Papers on Self-Study and Institutional Improvement, 2(2), 48-55. 
Zajceva, N.A. (2015). Generation theory: are we different or the same? Rossijskie regiony: vzglyad v budushchee, 2(3), 220-236. 